Check for updates

Cite this: Phys. Chem. Chem. Phys. 2021, 23, 16837

Received 28th April 2021 Accepted 13th July 2021

DOI: $10.1039 / d 1 c p 01863 a$

rsc.li/pccp

\title{
Scouting for strong light-matter coupling signatures in Raman spectra $\uparrow$
}

\author{
Wassie Mersha Takele, ${ }^{a b}$ Lukasz Piatkowski, (D) *c Frank Wackenhut, (D) *b \\ Sylwester Gawinkowski, (D) a Alfred J. Meixner (D) *b and Jacek Waluk (D)*ad
}

\begin{abstract}
Strong coupling between vibrational transitions and a vacuum field of a cavity mode leads to the formation of vibrational polaritons. These hybrid light-matter states have been widely explored because of their potential to control chemical reactivity. However, the possibility of altering Raman scattering through the formation of vibrational polaritons has been rarely reported. Here, we present the Raman scattering properties of different molecules under vibrational strong coupling conditions. The polariton states are clearly observed in the IR transmission spectra of the coupled system for benzonitrile and methyl salicylate in liquid phase and for polyvinyl acetate in a solid polymer film. However, none of the studied systems exhibits a signature of the polariton states in the Raman spectra. For the solid polymer film, we have used cavities with different layer structures to investigate the influence of vibrational strong coupling on the Raman spectra. The only scenario where alterations of the Raman spectra are observed is for a thin Ag layer being in direct contact with the polymer film. This shows that, even though the system is in the vibrational strong coupling regime, changes in the Raman spectra do not necessarily result from the strong coupling, but are caused by the surface enhancement effects.
\end{abstract}

\section{Introduction}

Molecular vibrations (MVs) can be excited either through the absorption or the inelastic scattering of photons. ${ }^{1}$ The former has been used to detect vibrations associated with a change of the dipole moment, whereas the latter, which is the basis of Raman spectroscopy, has been employed to probe vibrations involving a change in polarizability. ${ }^{1,2}$ Due to simple sample preparation, non-destructive nature and microscopy capabilities, Raman spectroscopy has become a valuable analytical tool. ${ }^{3-6}$ However, since MVs exhibit extremely small scattering crosssections, Raman scattering is a very inefficient process. As a result, Raman signals from low concentration samples are hard to detect and can, for resonance Raman, be overwhelmed by the molecular fluorescence signal. ${ }^{7-9}$ To circumvent this drawback, a

\footnotetext{
${ }^{a}$ Institute of Physical Chemistry, Polish Academy of Sciences, Kasprzaka 44/52, 01-224 Warsaw, Poland.E-mail: jwaluk@ichf.edu.pl

${ }^{b}$ Institute of Physical and Theoretical Chemistry and LISA+, University of Tübingen, Auf der Morgenstelle 18, D-72076 Tübingen, Germany.

E-mail: frank.wackenhut@uni-tuebingen.de, alfred.meixner@uni-tuebingen.de

${ }^{c}$ Faculty of Materials Engineering and Technical Physics, Poznan University of Technology, Piotrowo 3, 60-965 Poznań, Poland.

E-mail: lukasz.j.piatkowski@put.poznan.pl

${ }^{d}$ Faculty of Mathematics and Science, Cardinal Stefan Wyszyński University, Dewajtis 5, 01-815 Warsaw, Poland

$\dagger$ Electronic supplementary information (ESI) available. See DOI: 10.1039/ d1cp01863a
}

variety of enhancement strategies have been proposed over the years. The widely used method is surface-enhanced Raman spectroscopy (SERS) that increases Raman scattering by molecules adsorbed on, or located near metallic nanostructures or surfaces. ${ }^{8-11}$ Additionally, the fluorescence emission of the molecule is quenched due to the presence of the noble metal surface, which helps to detect the Raman scattering signal. ${ }^{12}$ In tip-enhanced Raman spectroscopy, significant Raman signal enhancement and better resolution can be obtained by combining the sensitivity of SERS and the lateral resolution of scanning probe microscope. ${ }^{13-15}$ The stimulating effect of vacuum fluctuations within a high-finesse microcavity (the Purcell Raman enhancement) has also been employed to improve Raman scattering from various kinds of materials, such as carbon nanotubes, ${ }^{16}$ a quantum well, ${ }^{17}$ atmospheric gases, ${ }^{18-21}$ and organic molecules. $^{22}$ This approach has a potential for miniaturization, as it may be incorporated in a spectrometer. ${ }^{18}$ Another route that has been explored to enhance Raman scattering efficiency is the strong light-matter coupling phenomenon. ${ }^{23-28}$

Strong coupling occurs when the coherent energy exchange between molecules and a microcavity ${ }^{29}$ or plasmon modes ${ }^{30}$ becomes faster than any competing dissipation process. It leads to the formation of half-light/half-matter quasiparticles termed polaritons. ${ }^{31}$ So far, polariton states formed by the coupling between excitons and the cavity ${ }^{23-25}$ or surface plasmon $^{26-28}$ modes have been widely investigated in terms of enhancing the Raman scattering process. In this polariton-mediated 
(a)

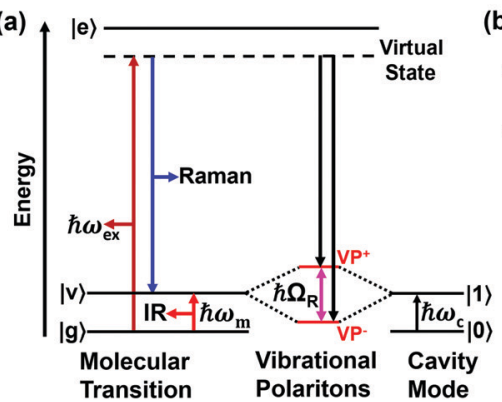

(b)

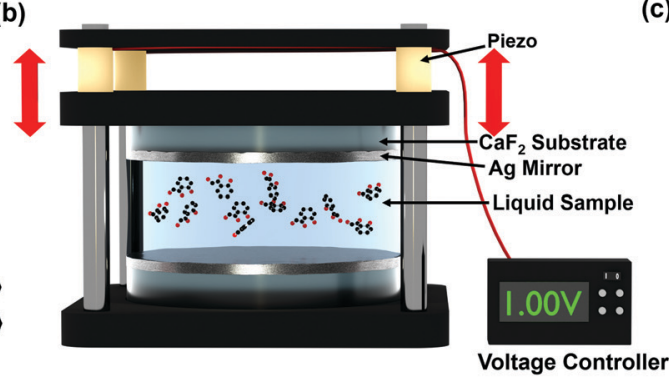

(c)

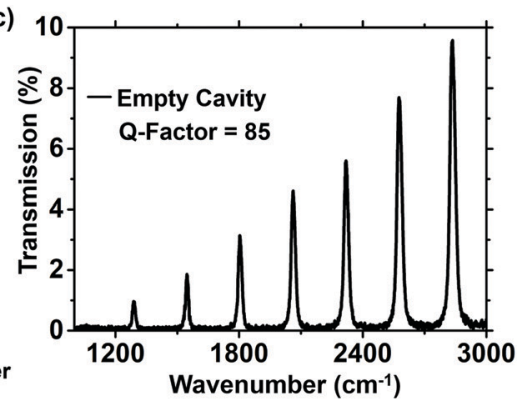

Fig. 1 (a) Simplified diagram illustrating the Raman scattering process and IR absorption of molecules under vibrational strong coupling. When the IR-vibrational frequency of the molecule $\left(\omega_{m}\right)$ coincides with the cavity mode $\left(\omega_{c}\right)$, vibrational polaritons $\left(V^{+}\right.$and $\left.V^{-}\right)$can be formed and the molecule in the virtual state may decay to either $\mathrm{VP}^{+}$or $\mathrm{VP}^{-}$mode (black arrows). (b) Schematic illustration of the open IR-Fabry-Pérot type microcavity structure used to couple liquid phase samples. (c) Transmission spectrum of an empty microcavity with a $Q$-factor of 85.

enhancement mechanism, maximum intensity is observed when the Raman excitation energy becomes resonant with polariton states. ${ }^{19,23}$ An alternative scheme proposed to increase the Raman scattering cross-section is vibrational strong coupling (VSC), ${ }^{32-34}$ which is realized when dipole active MVs strongly couple with the vacuum electric field of a microcavity. ${ }^{35-43}$

VSC results in the formation of vibrational polaritons $\left(\mathrm{VP}^{+}\right.$ and $\left.\mathrm{VP}^{-}\right)$, separated by the Rabi splitting energy ( $\hbar \Omega_{\mathrm{R}}$, Fig. 1a). In 2015, Shalabney et al. ${ }^{35}$ and Long and Simpkins ${ }^{36}$ independently showed the formation of vibrational polaritons by coupling the $\mathrm{C}=\mathrm{O}$ stretching modes of polyvinyl acetate and polymethyl methacrylate films with Fabry-Pérot cavity modes, respectively. Since then, VSC has been demonstrated for many systems such as neat liquids, ${ }^{38,39}$ solutions, ${ }^{40}$ ionic liquid crystals, ${ }^{41}$ protein vibrational modes ${ }^{42}$ and multimode vibrational transitions of a polymer. ${ }^{43}$ Experimentally, the $\mathrm{VP}^{+}$and $\mathrm{VP}^{-}$states can be observed in the transmission spectrum of the coupled system, as two new peaks with energies shifted from the original frequency by half the Rabi splitting. ${ }^{37,38}$ These hybrid light-matter states have attracted considerable attention because of their potential to control chemical reactivity, ${ }^{44-49}$ vibrational energy transfer, ${ }^{50}$ and nonlinear optical responses. ${ }^{51-53}$ Since Raman spectra directly reflect vibrational modes of the molecule, it is only natural to expect $\mathrm{VP}^{+}$and $\mathrm{VP}^{-}$states to be observed in Raman spectra. Indeed, Shalabney et $\mathrm{al}^{32}$ investigated the Raman scattering properties of vibrational polaritons obtained from a polymer film. In the experiment, the $\mathrm{C}=\mathrm{O}$ IR-vibration of polyvinyl acetate (PVAc) was coupled with the optical mode of the cavity. The observed modifications in the Raman spectra were attributed to the strong coupling and to the coherent nature of the vibrational polaritonic states, which enhances the Raman cross-section.

In this work, we investigate the effects of VSC on the Raman scattering properties of three systems: two organic compounds in a liquid phase and a solid polymer film. For the liquid phase samples, benzonitrile (BN) and methyl salicylate (MS), the experiments were realized by coupling the $\mathrm{C}=\mathrm{O}$ and $\mathrm{C} \equiv \mathrm{N}$ Raman vibrational mode with the vacuum cavity field of an open IR-microcavity. ${ }^{54}$ Additionally, we study the Raman scattering characteristics of a solid PVAc film under VSC. In order to obtain a complete picture of Raman VSC, we utilize different cavity geometries, which were prepared by varying the thickness and sequence of the cavity layers. Alterations in Raman spectra are observed only for cavities that support SERS effects. We did not find any signatures of VSC that would be consistent with the VSC observed in transmission spectra. Consequently, we find that it is crucial to not only consider VSC, but also other aspects, such as SERS, to understand lightmatter interaction in such systems. This observation is critical for a deeper understanding of the nature of strong light matter coupling and potential applications of Raman VSC in polaritonic chemistry to actively influence chemical reactivity.

\section{Materials and methods}

Methyl salicylate (99\%), methylcyclohexane (99\%), benzonitrile (99\%), toluene (99\%), and polyvinyl acetate (MW: 100 000) were purchased from Sigma-Aldrich and used without further purification. $\mathrm{CaF}_{2}$ windows (diameter: $25.0 \mathrm{~mm}$, thickness: $5.0 \mathrm{~mm}$ ) were purchased from Crystran LTD, while a mirror mount with a piezoelectric adjuster and a voltage controller were purchased from Thorlabs, Inc.

\section{Open microcavity preparation ${ }^{54,55}$}

The Ag films, typically $10 \mathrm{~nm}$ thick (unless otherwise explicitly stated), were deposited on a $\mathrm{CaF}_{2}$ substrate by a high vacuum sputter coater (Leica EM MED 020 coating system). The thickness of the metal film was controlled using a quartz microbalance film thickness monitoring system (EM QSG100) as a reference during sputtering. The microcavity was realized by bringing the Ag-coated $\mathrm{CaF}_{2}$ windows in close proximity and by carefully controlling their parallelism with the kinematic mirror holders. Subsequently, the cavity was filled by adding a few droplets of an appropriate solution onto the edges of the $\mathrm{CaF}_{2}$ windows using a micropipette. The solution flowed into the cavity by capillary action and remained stable between the mirrors during the entire experiment.

\section{Polymer spaced microcavity and SERS sample preparation}

Polyvinyl acetate (PVAc), 15\% w/w, was dissolved in toluene and the solution was sonicated at $35{ }^{\circ} \mathrm{C}$ for 30 minutes. After cooling to room temperature, the solution was filtered using 
a $0.22 \mu \mathrm{m}$ Nylon filter. To prepare the PVAc spaced cavity, $10 \mathrm{~nm}$ or $30 \mathrm{~nm} \mathrm{Ag}$ film (depending on the experiment) was deposited on $\mathrm{a} \mathrm{CaF}_{2}$ window. Then the PVAc solution was spincoated (Chetmat Technology INC.) at a speed of $870 \mathrm{rpm}$ for 60 s. These spinning conditions yielded a PVAc layer of approximately $5 \mu \mathrm{m}$ thickness and ensured that one of the cavity resonances was ON resonance with the PVAc carbonyl stretch mode. Finally, an optical microcavity was realized when another $10 \mathrm{~nm}$ thick $\mathrm{Ag}$ film (or different when explicitly stated) was deposited on top of the PVAc film. The OFF resonance cavity was obtained by spin-coating the same PVAc solution at a speed of $1000 \mathrm{rpm}$ for a duration of $60 \mathrm{~s}$. Multiple ON and OFF resonance samples were made and the preparation procedure proved to be highly reproducible.

In order to prepare the SERS sample, a few droplets of a colloidal silver nanoparticle solution (spheres with a diameter of $\sim 100 \mathrm{~nm}$ ) were cast on a clean $\mathrm{CaF}_{2}$ window and left until dry. Finally, a PVAc solution was deposited on top of the SERS substrate using a micropipette and spin-coated at a speed of $870 \mathrm{rpm}$ for a duration of $60 \mathrm{~s}$.

\section{Optical measurements}

All transmission spectra were recorded using a Fourier transform infrared (FTIR) spectrometer (Bruker Vertex 70). The spectra were measured with a resolution of $0.5 \mathrm{~cm}^{-1}$ and averaged from 50 scans. MS solutions $(0.05,0.5,5,15$ and $25 \% \mathrm{v} / \mathrm{v})$ were prepared by dissolving MS in methylcyclohexane. The transmission spectra of neat MS and neat BN were obtained by injecting the liquid between two uncoated $\mathrm{CaF}_{2}$ windows.

Prior to each vibrational strong coupling experiment, the quality of an empty cavity was verified by measuring its transmission spectrum. Then the cavity was filled with a solution and a voltage applied to the piezo element to tune the cavity mode towards the frequency of the molecular vibration under study. To measure FTIR spectra of the uncoupled, reference polymer sample, a PVAc film ( $5 \mu \mathrm{m}$ thick) was spin-coated on a clean $\mathrm{CaF}_{2}$ window. For all systems considered, the Rabi splitting, i.e., the separation between the upper and lower polaritonic peak, was determined when the cavity mode was isoenergetic with the molecular vibration of interest. Raman scattering and SERS measurements were performed using Renishaw inVia micro-Raman system. For all measurements, the laser excitation wavelength was set to $532 \mathrm{~nm}$. For the MS and BN filled open microcavity, the excitation laser light was focused onto the sample surface using a long working distance Renishaw $15 \times$ objective. Raman spectra of the PVAc-spaced microcavities and the SERS sample were collected with a $50 \times$ Leica microscope objective (NA $=0.75$ ). A movable stage was employed to move the sample and acquire 2D mapping. Data were collected in a raster scanning fashion, and the final spectra were obtained by averaging at least 140 spectra acquired from a large area of the microcavity.

\section{Results}

Fig. 1a illustrates the concept of the Raman scattering process under VSC. When a molecule is irradiated with monochromatic light with a photon energy $\hbar \omega_{\text {exc }}$, it will be transferred from the ground electronic state to an excited nonstationary virtual state. In the normal Stokes Raman scattering process, the molecule in the virtual state relaxes into the first excited vibrational state (blue arrow). However, if the first excited vibrational state is strongly coupled to the cavity mode, the molecule in the virtual state may decay to either the $\mathrm{VP}^{+}$or $\mathrm{VP}^{-}$mode (black arrows in Fig. 1a). ${ }^{32-34}$ In ref. 54, we have used a piezo-based tunable Fabry-Pérot type open microcavity to investigate infrared VSC of organic molecules and Fig. 1b shows the structure of the microcavity used in our experiments. The cavity consists of two $\mathrm{CaF}_{2}$ windows coated with $\mathrm{Ag}$ metal film. The two mirrors were mounted into mirror holders and the optical path length $\left(L_{\mathrm{op}}\right)$ of the cavity was tuned by applying a voltage to the piezoelectric stacks integrated into one of the mirror mounts. An exemplary transmission spectrum of an empty IR-microcavity measured at normal incidence is shown in Fig. 1c. The long optical path length of the cavity $\left(L_{\mathrm{op}} \sim 20 \mu \mathrm{m}\right)$ is reflected in a relatively small free spectral range $\left(\mathrm{FSR}=260 \mathrm{~cm}^{-1}\right)$ and the multiple cavity resonances are present in the shown spectral range. ${ }^{51}$ The decrease of the cavity transmission towards lower wavenumbers is caused by the evolution of the dielectric constant of the Ag film in this spectral region. ${ }^{38}$ The transmission spectra of an empty cavity as a function of the voltage applied to the piezo actuators are shown in the Fig. S1 (ESI $\dagger$ ).

First, we studied the Raman scattering properties of benzonitrile (BN) under VSC. The $\mathrm{C} \equiv \mathrm{N}$ mode of $\mathrm{BN}$ is well separated from other vibrational modes and it is both IR and Raman active (blue curves in Fig. 2a and b, respectively). Vibrational polaritons were observed in the neat, liquid $\mathrm{BN}$, when the $\mathrm{C} \equiv \mathrm{N}$ IR stretching frequency at $2229 \mathrm{~cm}^{-1}$ was hybridized with one of the cavity modes (Fig. 2a, red curve). The separation between the $\mathrm{VP}^{+}$and $\mathrm{VP}^{-}$peaks - which, in resonant condition, is equivalent to the Rabi splitting - is found to be $50 \mathrm{~cm}^{-1}$, and it is comparable to the Rabi splitting values of the $\mathrm{C} \equiv \mathrm{N}$ transition in $\mathrm{BN}\left(54 \mathrm{~cm}^{-1}\right)^{38}$ and 4-butylbenzonitrile $\left(46 \mathrm{~cm}^{-1}\right) .{ }^{56,57}$ The value of the Rabi splitting is much larger than the linewidth of the cavity mode $\left(\sim 22 \mathrm{~cm}^{-1}\right)$ and the molecular resonance $\left(\sim 12 \mathrm{~cm}^{-1}\right)$. This confirms that coupling between the cavity mode and the $\mathrm{C} \equiv \mathrm{N}$ transition in $\mathrm{BN}$ satisfies the criterion of vibrational strong coupling. ${ }^{38}$ In Fig. 2a (black curve) splitting into lower $\left(\mathrm{VP}^{-}\right)$and upper $\left(\mathrm{VP}^{+}\right)$vibrational polaritons were not clearly observed in the spectrum of BN filled microcavity, as the cavity resonance was strongly detuned from $\mathrm{C} \equiv \mathrm{N}$ stretching of $\mathrm{BN}$. The minute dispersive line shape feature observed at about $2220 \mathrm{~cm}^{-1}$ is associated with the weak highly detuned cavity-molecule interaction. This sample is considered as an OFF resonant cavity, due to the absence of the two polaritonic states in the coupled system.

The Raman spectra of BN inside the ON (red) and OFF (black) resonant microcavity are shown in Fig. 2b, together with a reference spectrum (blue curve) acquired in free space. Even though the Ag cavity mirrors decrease the intensity, new peaks due to the vibrational polaritons were not observed in the ON resonance case. Instead, the acquired Raman spectrum exhibits 
(a)

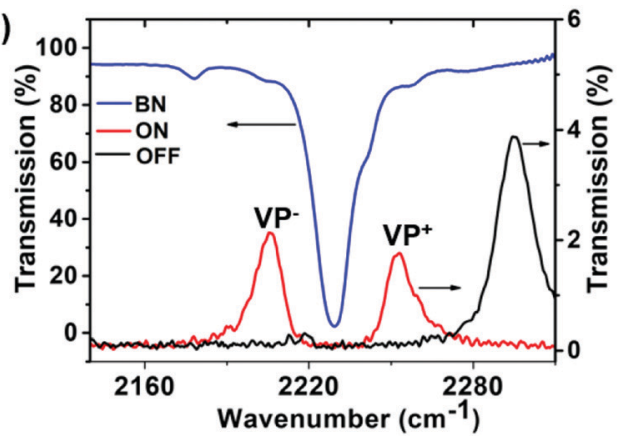

(c)

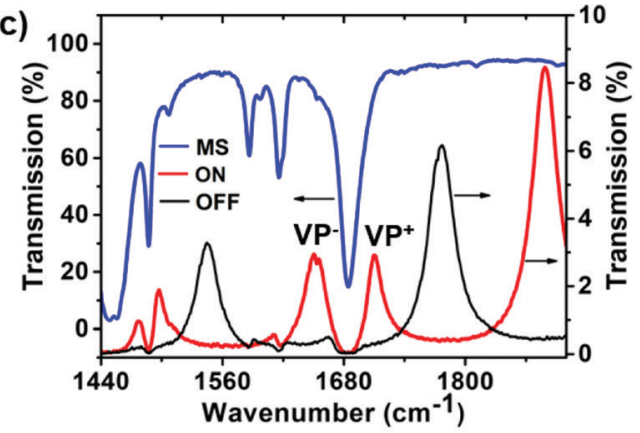

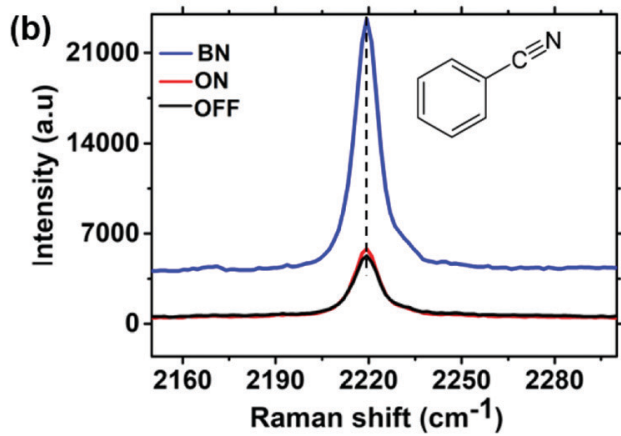

(d)

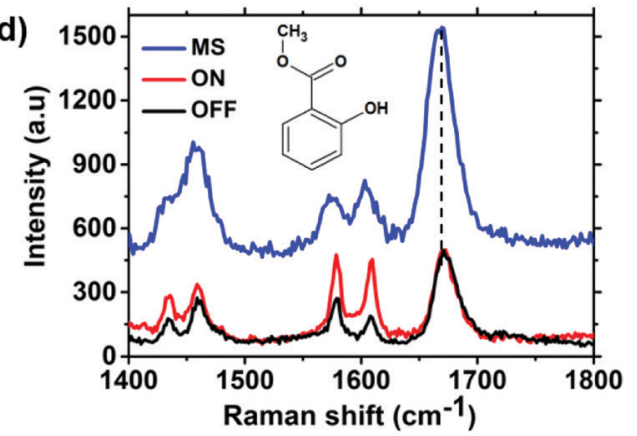

Fig. 2 (a) Transmission spectra of ON (red) and OFF (black) resonance microcavities filled with benzonitrile. The FTIR spectrum of uncoupled benzonitrile is shown in blue. (b) Raman spectra of BN obtained from the ON (red) and OFF (black) resonance cavities shown in panel (a). The Raman spectrum of BN from the reference sample is shown in blue, the inset shows the molecular structure of BN. (c) FTIR spectrum of $15 \%$ v/v MS dissolved in methylcyclohexane (blue). The red and black curves show the transmission spectrum of the solution placed inside ON and OFF resonance microcavity, respectively. (d) Raman spectrum of MS from the ON (red) and OFF (black) resonance cavities shown in panel c, the inset shows the molecular structure of MS.

a single vibrational band identical with that observed for the reference sample. This observation suggests that Raman scattering by the dominant fraction of the probed oscillators is insensitive to VSC and vibrational polariton peaks observed in the FTIR measurements cannot be probed using Raman scattering.

Fig. $2 \mathrm{c}$ and $\mathrm{d}$ show the results obtained for methyl salicylate (MS), which has richer vibrational spectrum, exhibiting several IR and Raman active vibrational peaks (blue lines). The infrared transmission spectra of the MS-filled cavity, set to be ON (red) resonance with the MS carbonyl stretch mode at $1685 \mathrm{~cm}^{-1}$, are shown in Fig. 2c. When the cavity mode was tuned towards the carbonyl stretch resonance, new transitions at $1650 \mathrm{~cm}^{-1}$ and $1710 \mathrm{~cm}^{-1}$ could be observed in the transmission spectrum of the coupled system (red line), which has been described in detail by Takele et $a l .{ }^{54}$ These are the signatures of the vibrational hybrid light-matter states $\left(\mathrm{VP}^{-}\right.$and $\left.\mathrm{VP}^{+}\right)$in $\mathrm{MS}$. The presence of VSC in MS is verified by the polariton anticrossing behaviour as a function of cavity tuning (see Fig. S2, ESI $\dagger$ ). The transmission spectrum of another MS-filled cavity, in which the cavity mode was strongly detuned with respect to the $\mathrm{C}=\mathrm{O}$ stretch vibration of MS is displayed in Fig. 2c (black curve). The weak dispersive line shape observed at about $1665 \mathrm{~cm}^{-1}$ is caused by the weak interaction between the cavity mode at about $1780 \mathrm{~cm}^{-1}$ and the $\mathrm{C}=\mathrm{O}$ vibrational transition of MS. Due to the lack of the two polaritonic peaks, we consider this configuration as an OFF resonant sample.

The Raman spectrum of a reference MS sample, prepared by injecting the solution in-between two $\mathrm{CaF}_{2}$ windows without the $\mathrm{Ag}$ films, is depicted in Fig. $2 \mathrm{~d}$ (blue line). The $\mathrm{C}=\mathrm{O}$ vibration of MS is observed at $1670 \mathrm{~cm}^{-1}$, which is in a full agreement with the value reported before. ${ }^{58}$ Subsequently, Raman spectra were recorded from the MS-filled cavity under VSC conditions for a cavity resonance tuned to be ON or OFF resonant to $\mathrm{C}=\mathrm{O}$ vibration (Fig. $2 \mathrm{~d}$, red and black lines, respectively). Apart from an overall decrease of the Raman signal intensity (due to lower transmission of the semitransparent cavity mirrors) no changes or formation of new spectral features can be observed for the three scenarios shown in Fig. 2d. In order to ensure that the cavity structure is not altered during the Raman experiment, the infrared transmission spectrum of the coupled system was acquired before and after the Raman measurement to confirm that the sample remains under strong coupling conditions (see Fig. S3, ESI $\dagger$ ). Furthermore, the cavity assembly was scanned in the focal plane to verify that the measured spectra remain unchanged irrespectively of the position probed with the laser.

The experiments shown in Fig. 2 were all carried out in liquid phase and did not show any signature of Raman strong coupling. The third system investigated here is a PVAc film, which is used as a spacer between the cavity mirrors with a suitable thickness to support resonances in the IR spectral region. As depicted in Fig. 3a (black line), the PVAc layer has an intense IR peak at $1740 \mathrm{~cm}^{-1}$ due to the stretching vibration of the $\mathrm{C}=\mathrm{O}$ group. When the cavity transmission maximum was tuned toward the $\mathrm{C}=\mathrm{O}$ transition of PVAc, the infrared transmission spectrum of the polymer loaded cavity showed two 
distinct peaks at 1650 and $1803 \mathrm{~cm}^{-1}$. These are lower $\left(\mathrm{VP}^{-}\right)$ and upper $\left(\mathrm{VP}^{+}\right)$vibrational polaritons in PVAc (Fig. 3a, red line). The observed Rabi splitting $\left(153 \mathrm{~cm}^{-1}\right)$ is much larger than the bandwidth of both the cavity mode $\left(45 \mathrm{~cm}^{-1}\right)$ and the $\mathrm{C}=\mathrm{O}$ vibration of PVAc $\left(30 \mathrm{~cm}^{-1}\right)$. This confirms that the hybridized system fulfills the criterion of strong coupling, i.e., the coupling strength is larger than the bandwidths of the cavity mode and the molecular transition. ${ }^{30}$ The green line in Fig. 3a shows the transmission spectrum of another PVAc loaded cavity, which was prepared by varying only the PVAc thickness during spin coating (for details, see Methods). This sample configuration was considered to be an OFF resonant cavity, as vibrational polariton signatures were not clearly observed. The small residual peak observed at $1707 \mathrm{~cm}^{-1}$ is due to a weak interaction between the higher order cavity mode at $1940 \mathrm{~cm}^{-1}$ and the $\mathrm{C}=\mathrm{O}$ vibrational transition of PVAc film.

The Raman spectrum of a PVAc film on a bare $\mathrm{CaF}_{2}$ substrate (configuration S1 in Fig. 3e) is presented by the black line in Fig. $3 \mathrm{~b}$ and serves as reference. The Raman spectrum shown in red in Fig. 3b was acquired from an $\mathrm{ON}$ resonance cavity, where the mirrors are formed by two $10 \mathrm{~nm} \mathrm{Ag}$ layers (MC1 in Fig. 3e). In this case, the spectral shape and intensity of the Raman spectrum is strongly altered compared to the reference spectrum of sample $\mathrm{S} 1$ and the largest differences (a)

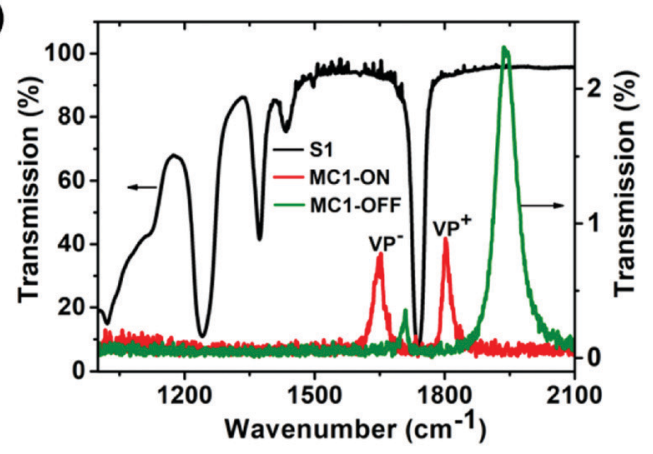

(c)

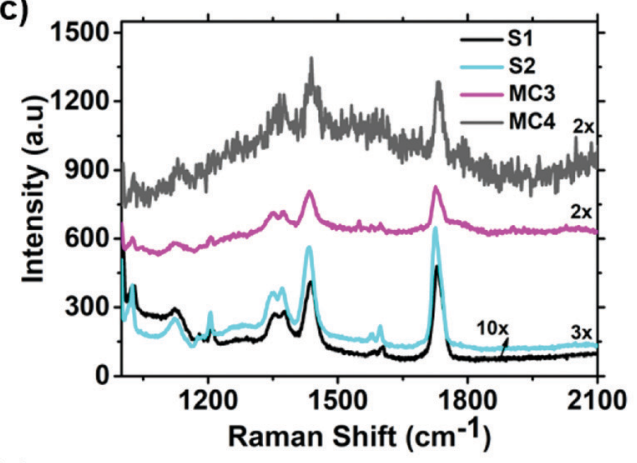

(b)

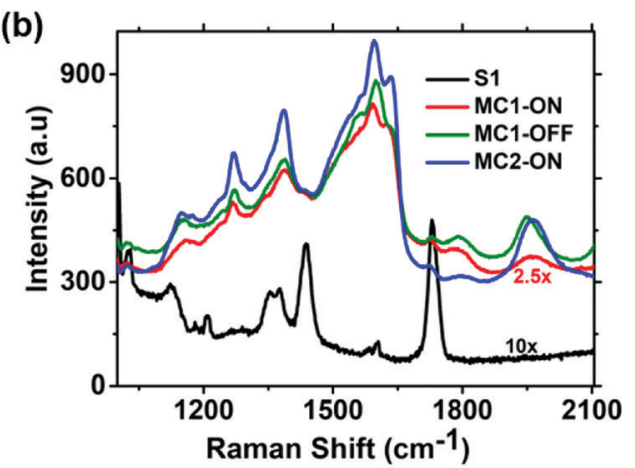

(d)

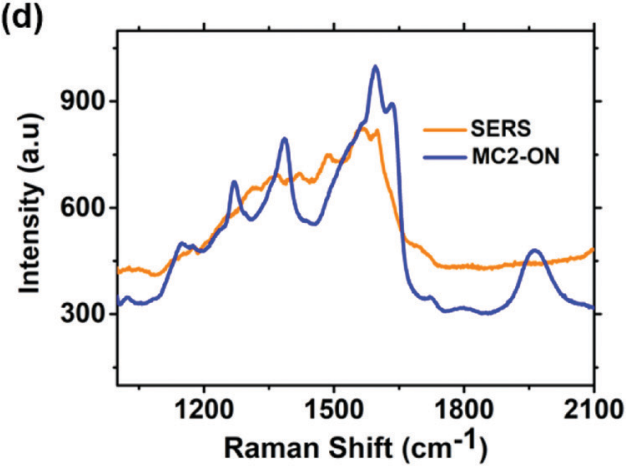

(e)
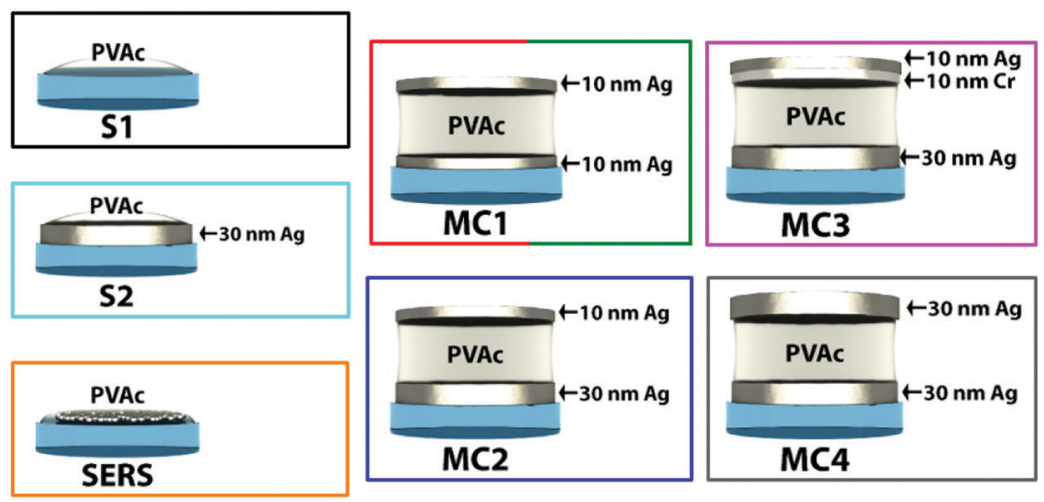

Fig. 3 (a) Infrared transmission of a reference PVAc film ( $\mathrm{S} 1$ in panel (e)) deposited on a $\mathrm{CaF}_{2}$ window (black line) and embedded in an optical microcavity ( $M C 1$ in panel (e)), which is ON (red line) or OFF (green line) to the $\mathrm{C}=\mathrm{O}$ vibration of PVAc. (b) Corresponding Raman spectra of the configurations shown in (a). Additionally, the Raman spectrum of configuration MC2 is shown in blue. (c) Raman spectra of PVAc obtained with different sample configurations shown schematically in panel (e); for MC3 and MC4 the measurements were conducted under resonance condition. (d) Comparison of the SERS spectrum of PVA acquired using $100 \mathrm{~nm}$ spherical Ag nanoparticles as a substrate and the Raman spectrum obtained from configuration MC2. (e) Schematic illustration of different sample configurations; S1, S2, and SERS indicate a thin PVAc film deposited on a CaF 2 window, a $30 \mathrm{~nm}$ Ag film, and a SERS substrate, respectively. MC1 to MC4 illustrate different PVAc spaced microcavity configurations. 
can be observed in the spectral regions around $1600 \mathrm{~cm}^{-1}$ and $1950 \mathrm{~cm}^{-1}$. Similar spectral changes were reported by Shalabney et al. and were assigned to Raman VSC. ${ }^{32}$ Interestingly, we observed the same spectral features for an OFF resonant MC1 cavity with two $10 \mathrm{~nm} \mathrm{Ag}$ layers (Fig. 3b, green spectrum), even though the infrared transmission spectrum clearly indicates the absence of strong coupling (Fig. 3a, green spectrum). The infrared transmission spectrum of the cavity configuration MC1, acquired before and after the Raman measurement, is shown in the ESI $\dagger$ (Fig. S4) and is not influenced by the Raman experiment. The appearance of similar spectral features for the ON and OFF resonant case indicates that they cannot be associated with the formation of polaritonic states. However, to reproduce the experimental conditions used by Shalabney et al., ${ }^{32}$ we have replaced the bottom layer by a $30 \mathrm{~nm}$ thick $\mathrm{Ag}$ layer and kept the top layer constant at $10 \mathrm{~nm}$ (MC2 in Fig. 3e). The resulting Raman spectrum is presented in Fig. 3b (blue line) and the spectral shape is practically the same as already observed for MC1. This observation suggests that an increase of the bottom layer thickness has no significant impact on the overall spectral shape and the Raman spectra are strongly altered in the cavity configurations MC1 and MC2. However, the Raman spectra of the cavities MC1/MC2 are significantly more intense compared to the reference spectrum obtained from the reference sample $\mathrm{S} 1$, suggesting that surface enhancement effects may play a role. To verify whether surface effects have any influence on the intensity and spectral shape, we have acquired a series of Raman spectra using different sample configurations (S2, MC3, and MC4). The first configuration is a PVAc film directly spin-coated on a $30 \mathrm{~nm} \mathrm{Ag}$ layer without a top mirror (S2 in Fig. 3e), which is shown in cyan in Fig. 3c. This Raman spectrum is similar to the reference spectrum of sample S1 and the spectral features observed for $\mathrm{MC1} / \mathrm{MC} 2$ are not present. This suggests that a single $30 \mathrm{~nm}$ thick $\mathrm{Ag}$ layer does not induce the changes observed in Fig. 3b. The next step is to prevent direct contact between the top Ag layer and the PVAc film. This is achieved in the cavity configuration MC3 (Fig. 3e) by introducing a $10 \mathrm{~nm}$ thick Cr film to separate the polymer and the $\mathrm{Ag}$ layer. The infrared transmission spectrum (Fig. S5, ESI $\dagger$ ) of this configuration confirms that the cavity and the $\mathrm{C}=\mathrm{O}$ vibration are still strongly coupled. Interestingly, the shape of the PVAc Raman spectrum (Fig. 3c, purple) using the cavity MC3 is the same as the reference spectrum of sample S1 (Fig. 3c, black), indicating that the increased intensity and altered spectral shape in Fig. 3b is caused by direct contact between the $\mathrm{Ag}$ layer and the PVAc film. The last cavity configuration is MC4, where the thickness of both $\mathrm{Ag}$ layers is increased to $30 \mathrm{~nm}$ and the respective Raman spectrum is shown in Fig. 3c (grey line). Obviously, in this case, the Raman spectrum is more noisy, due to lower transmission of the cavity mirrors. Nevertheless, the main spectral features are still visible and closely resemble the reference spectrum of sample $\mathrm{S} 1$.

These results show that the PVAc Raman spectra of the configurations shown in Fig. $3 \mathrm{c}$ are identical to that of the reference sample $\mathrm{S} 1$ and the only ones differing from the reference are those with an unprotected $10 \mathrm{~nm} \mathrm{Ag}$ layer. Evidently, a thin silver layer exhibits some degree of percolation and supports surface enhancement. The surface roughness and nanometer-scale structure of the cavity mirrors used in our study have been characterized by AFM (Fig. S6, ESI $\dagger$ ) and show that their morphology is different, possibly leading to different SERS enhancements. However, if the spectral patterns of the MC1 and MC2 cavities indeed originate from surface enhancement effects, then acquiring Raman spectra from PVAc spin-coated on a typical SERS substrate should yield similar spectra. To this end, we acquired Raman spectra (or rather SERS spectra) of PVAc spincoated on a surface made of a layer of $100 \mathrm{~nm} \mathrm{Ag}$ nanospheres (SERS in Fig. 3e). The measured PVAc SERS spectrum indeed closely resembles that of the MC1 (ON and OFF resonance) and MC2 samples - the comparison is presented in Fig. 3d.

PVAc exhibits Raman bands at $1023 \mathrm{~cm}^{-1}, 1375 \mathrm{~cm}^{-1}, 1440 \mathrm{~cm}^{-1}$ and $1730 \mathrm{~cm}^{-1}$, which are assigned to the $\mathrm{CH}_{2}$ twisting, $\mathrm{CH}_{3}$ asymmetric deformation, $\mathrm{CH}_{2}$ asymmetric deformation, and $\mathrm{C}=\mathrm{O}$ stretching, respectively. ${ }^{59,60}$ All these vibrations are clearly visible in the Raman spectrum of neat PVAc shown in Fig. 4a (black line), but none of these peaks is suitable to explain the appearance of the strong SERS peaks in Fig. $3 \mathrm{~b}$ in the spectral region at approximately $1600 \mathrm{~cm}^{-1}$. However, additional weak Raman peaks at $1580 \mathrm{~cm}^{-1}$ and $1600 \mathrm{~cm}^{-1}$ are present in the spectrum of the reference sample S1 (Fig. 4b, red line), which is prepared by spin coating PVAc dissolved in toluene. These peaks could be the origin of the spectral features observed in Fig. $3 \mathrm{~b}$ and are most probably caused by residuals of toluene left in the PVA film after evaporation, since the peak positions are consistent with the Raman spectrum of liquid toluene (Fig. 4c, blue line). ${ }^{61,62}$ These peaks have been assigned to phenyl stretching modes of toluene ${ }^{61-63}$ and can be strongly enhanced by SERS (Fig. 4d, pink line). ${ }^{61}$ Interestingly, the SERS spectrum of toluene (Fig. 4d, pink line) and the Raman spectrum of PVAc film dissolved in toluene obtained using cavity configuration MC2 (Fig. 4e, green line) show a fair agreement between their spectral features. This suggests that

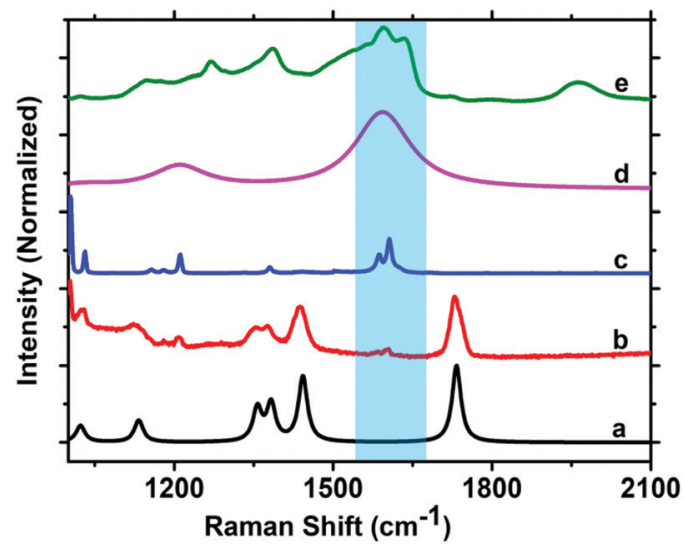

Fig. 4 Comparison of Raman spectra of PVAc and toluene: neat PVAc (black, a), PVAc film dissolved in toluene (red, b), liquid toluene (blue, c), SERS spectrum of liquid toluene (purple, d), and PVAC in MC2 cavity configuration shown in Fig. 3e (green, e). The Raman spectrum of the neat PVAc powder is from Taylor et al., ${ }^{59}$ whereas the SERS spectrum of liquid toluene is obtained from Qian et al. ${ }^{61}$ 
the strong, broad band observed at approximately $1600 \mathrm{~cm}^{-1}$ for the cavities MC1/MC2 is likely caused by SERS enhancement of phenyl stretching vibrations of toluene.

\section{Discussion and conclusions}

Experimental and theoretical work where VSC has been employed to alter Raman scattering are rather scarce. To the best of our knowledge, only Shalabney et $a .^{32}$ claimed enhancement of the Raman signal due to the formation of vibrational polaritons, particularly two to three orders of magnitude enhancement from the $\mathrm{VP}^{-}$state. The authors explained their observation by an increase of the total Raman cross-section due to strong coupling of the $\mathrm{C}=\mathrm{O}$ vibration of a PVAc polymer film with the cavity mode. On the other hand, Pino et al. ${ }^{33}$ employed a series of complex theoretical models and showed that the total Raman cross-section is conserved during VSC, as long as ultrastrong VSC, the regime characterized by a Rabi splitting comparable to the vibrational frequency, ${ }^{64}$ is not reached. They showed that the induced change in the ground state via ultrastrong VSC can lead to a modest enhancement of Raman scattering by less than a factor of two. Additionally, they found that the Stokes Raman and IR peaks in strongly coupled systems are located at the same energies, thus the same Rabi splitting energy should be observed in the Raman and in the infrared transmission spectra. This is in contrast to experimental results by Shalabney et al., ${ }^{32}$ where the Rabi splitting energy obtained from the Raman data was about two times larger than the one obtained from the IR transmission spectrum. Strashko and Keeling ${ }^{34}$ theoretically predicted significant enhancement of Raman scattering under ultrastrong VSC. On the other hand, it has been reported by many researchers that strong coupling of a material's electronic state with a microcavity or localized surface plasmon modes leads to modification of Raman scattering efficiency. ${ }^{23-28}$ For instance, Tartakovskii et $a .^{23}$ showed a significant Raman signal enhancement from a microcavity containing cyanine dye J aggregates, when both the excitation energy and scattered photons are in resonance with the polaritonic states. Nagasawa et $a .^{27}$ demonstrated the influence of strong coupling between silver nanodimer plasmon modes and excitons of hexamethylindotricarbocyanine perchlorate molecules on the Raman scattering properties of the molecules. They obtained maximum Raman enhancement at the resonance energy between the polariton states and the Raman excitation. Minamimoto et al. $^{28}$ also showed that electrochemically controlled strong coupling can be used to enhance Raman scattering spectra of molecules. In all of these and other works, ${ }^{24-26}$ neither polaritonic signatures nor a shift in the Raman peak positions have been observed in the Raman spectra of the coupled systems.

Here, we studied the possible effects of VSC on the Raman scattering properties of molecules. For the two liquid systems of MS and BN, we find no evidence for strong coupling in the Raman spectra, even though the IR transmission spectra show that the system is clearly strongly coupled. Additionally, the Raman spectra are the same regardless if the cavity is ON or OFF resonant to a Raman vibration. In contrast, the spectral shape and intensity of the Raman spectrum of a PVAc polymer film can be strongly altered by a microcavity. In some cases, new peaks can be observed and have been assigned to Raman VSC. ${ }^{32}$ However, these new peaks have different characteristics compared to the polaritonic peaks observed in the IR transmission spectrum. First, the full width at half maximum of the peaks in the Raman spectrum is larger compared to the $\mathrm{VP}^{+}$and $\mathrm{VP}^{-}$ peaks in the infrared transmission spectrum. Second, the separation between these peaks is unexpectedly large, two times larger than the Rabi splitting obtained from infrared data. These different characteristics already indicate that the origin of these peaks is not Raman strong coupling, since both the peak position and FWHM should be same in the Raman and infrared spectra. ${ }^{33}$ Comparing the broad and strong peaks observed at about $1600 \mathrm{~cm}^{-1}$ with literature values it is evident, that they do not emerge from the polaritonic states, but rather from the SERS enhancement of the phenyl vibrational peaks of toluene residuals. Nevertheless, coupling effects might still be present, but hidden by the strong SERS effect. However, the Raman spectrum obtained by separating the upper $\mathrm{Ag}$ mirror from the PVAc film by a thin Cr layer shows no observable difference compared with the reference spectrum of a pure PVAc film. This unequivocally proves that Raman scattering to the levels arising from vibrational strong coupling effect has not been detected. The hybrid molecule-cavity systems contain many uncoupled molecules, or the so-called "dark states", 65-67 which are molecular vibrations that are not interacting or interacting weakly with the cavity mode. This is mainly caused by a mismatch between the orientation of the transition dipole moment and the electric field in the cavity. ${ }^{53}$ In contrast to the polaritonic states, these "dark states" are invisible in the infrared transmission measurements. However, in the Raman measurement, the entire bulk sample is probed. Consequently, the signal from the strongly coupled molecules can be overwhelmed by scattering of uncoupled molecules, making the detection of polaritonic states highly challenging. In the future, additional experimental and theoretical works should be performed in order to quantitatively disentangle coupled/ uncoupled molecules. For instance, the effect of ultrastrong vibrational coupling on Raman scattering could be investigated. In this coupling regime, the coupling rate is similar to the vibrational frequency, and it was theoretically predicted that Rabi splitting could be observed in the Raman spectra. ${ }^{33,34}$

\section{Conflicts of interest}

There are no conflicts to declare.

\section{Acknowledgements}

W. M. T. acknowledges support from the European Union's Horizon 2020 research and innovation programme under the 
Marie Skłodowska-Curie grant agreement No. 711859 (CO-FUND NaMeS project) and the financial resources from Ministry of Science of Poland for science in the years 2017-2021 awarded for the implementation of an international cofinanced project. The project has received funding from the National Science Centre, Poland, grants 2019/35/B/ST4/00297 and 2017/26/M/ST4/00872, and from the European Union's Horizon 2020 research and innovation programme under the Marie Skłodowska-Curie grant agreement No. 665778. F. W. and A. J. M. acknowledge support from the DFG grand ME 1600/13-3. L. P. acknowledges the Installation Grant (EMBO) and the POIR.04.04.00-00-5D32/18-00 project carried out within the First TEAM programme of the Foundation for Polish Science co-financed by the European Union under the European Regional Development Fund. SG acknowledges support from the National Science Centre, Poland, grant 2018/29/B/ ST4/00089.

\section{References}

1 F. Siebert and P. Hildebrandt, Vibrational Spectroscopy in Life Sciences, WILEY-VCH Verlag GmbH and Co. KGaA, Weinheim, 2008.

2 W. E. Smith and G. Dent, Modern Raman Spectroscopy-A Practical Approach, John Wiley and Sons, Ltd, 2005.

3 H. J. Butler, L. Ashton, B. Bird, G. Cinque, K. Curtis, J. Dorney, K. Esmonde-White, N. J. Fullwood, B. Gardner, P. L. Martin-Hirsch, M. J. Walsh, M. R. McAinsh, N. Stone and F. L. Martin, Using Raman Spectroscopy to Characterize Biological Materials, Nat. Protoc., 2016, 11, 664-687.

4 Y. S. Li and J. S. Church, Raman Spectroscopy in the Analysis of Food and Pharmaceutical Nanomaterials, J. Food Drug Anal., 2014, 22, 29-48.

5 H. Kim, K. M. Kosuda, R. R. Van Duyne and P. C. Stair, Resonance Raman and Surface- and Tip-Enhanced Raman Spectroscopy Methods to Study Solid Catalysts and Heterogeneous Catalytic Reactions, Chem. Soc. Rev., 2010, 39, 4820-4844.

6 C. K. Muro, K. C. Doty, J. Bueno, L. Halámková and I. K. Lednev, Vibrational Spectroscopy: Recent Developments to Revolutionize Forensic Science, Anal. Chem., 2015, 87, 306-327.

7 R. R. Jones, D. C. Hooper, L. Zhang, D. Wolverson and V. K. Valev, Raman Techniques: Fundamentals and Frontiers, Nanoscale Res. Lett., 2019, 14, 2-34.

8 R. Panneerselvam, G. K. Liu, Y. H. Wang, J. Y. Liu, S. Y. Ding, J. F. Li, D. Y. Wu and Z. Q. Tian, SurfaceEnhanced Raman Spectroscopy: Bottlenecks and Future Directions, Chem. Commun., 2017, 54, 10-25.

9 J. Langer, D. J. de Aberasturi, J. Aizpurua, R. A. AlvarezPuebla, B. Auguié, J. J. Baumberg, G. C. Bazan, S. E. J. Bell, A. Boisen and A. G. Brolo, et al., Present and Future of Surface-Enhanced Raman Scattering, ACS Nano, 2020, 14, 28-117.
10 A. I. Pérez-Jiménez, D. Lyu, Z. Lu, G. Liu and B. Ren, SurfaceEnhanced Raman Spectroscopy: Benefits, Trade-Offs and Future Developments, Chem. Sci., 2020, 11, 4563-4577.

11 L. Piatkowski, J. T. Hugall and N. van Hulst, Raman Spectroscopy: Watching a Molecule Breathe, Nat. Photonics, 2014, 8, 589-591.

12 G. P. Acuna, M. Bucher, I. H. Stein, C. Steinhauer, A. Kuzyk, P. Holzmeister, R. Schreiber, A. Moroz, F. D. Stefani, T. Liedl, F. C. Simmel and P. Tinnefeld, Distance Dependence of Single-Fluorophore Quenching by Gold Nanoparticles Studied on DNA Origami, ACS Nano, 2012, 6, 3189-3195.

13 F. Shao and R. Zenobi, Tip-Enhanced Raman Spectroscopy: Principles, Practice, and Applications to Nanospectroscopic Imaging of 2D Materials, Anal. Bioanal. Chem., 2019, 411, 37-61.

14 D. Zhang, X. Wang, K. Braun, H. J. Egelhaaf, M. Fleischer, L. Hennemann, H. Hintz, C. Stanciu, C. J. Brabec, D. P. Kern and A. J. Meixner, Parabolic Mirror-Assisted Tip-Enhanced Spectroscopic Imaging for Non-Transparent Materials, J. Raman Spectrosc., 2009, 40, 1371-1376.

15 L. E. Hennemann, A. J. Meixner and D. Zhang, Surface- and Tip-Enhanced Raman Spectroscopy of DNA, Spectroscopy, 2010, 24, 119-124.

16 T. Hummer, J. Noe, M. S. Hofmann, T. W. Hansch, A. Hogele and D. Hunger, Cavity-Enhanced Raman Microscopy of Individual Carbon Nanotubes, Nat. Commun., 2016, 7, 1-7.

17 A. Fainstein, B. Jusserand and V. Thierry-Mieg, Raman Efficiency in a Planar Microcavity, Phys. Rev. B: Condens. Matter Mater. Phys., 1996, 53, R13287.

18 B. Petrak, J. Cooper, K. Konthasinghe, M. Peiris, N. Djeu, A. J. Hopkins and A. Muller, Isotopic Gas Analysis through Purcell Cavity Enhanced Raman Scattering, Appl. Phys. Lett., 2016, 108, 091107.

19 A. Fainstein and B. Jusserand, Raman Scattering in Resonant Cavities, Top. Appl. Phys., 2006, 108, 17-110.

20 B. Petrak, N. Djeu and A. Muller, Purcell-Enhanced Raman Scattering from Atmospheric Gases in a High-Finesse Microcavity, Phys. Rev. A: At., Mol., Opt. Phys., 2014, 89, 023811.

21 J. Gomez Velez and A. Muller, Purcell-Enhanced Microcavity Raman Scattering from Pressurized Gases, Appl. Phys. Lett., 2018, 112, 041107.

22 F. Cairo, F. D. Martini and D. Murra, QED-Vacuum Confinement of Inelastic Quantum Scattering at Optical Frequencies: A New Perspective in Raman Spectroscopy Spectroscopy, Phys. Rev. Lett., 1993, 70, 1413-1416.

23 A. I. Tartakovskii, M. Emam-Ismail, D. G. Lidzey, M. S. Skolnick, D. D. Bradley, S. Walker and V. M. Agranovich, Raman Scattering in Strongly Coupled Organic Semiconductor Microcavities, Phys. Rev. B: Condens. Matter Mater. Phys., 2001, 63, 121302.

24 A. Fainstein, B. Jusserand and V. Thierry-Mieg, CavityPolariton Mediated Resonant Raman Scattering, Phys. Rev. Lett., 1997, 78, 1576-1579. 
25 W. R. Tribe, D. Baxter, M. S. Skolnick, D. J. Mowbray, T. A. Fisher and J. S. Roberts, In- and out-going resonant Raman scattering from the cavity polaritons of semiconductor quantum microcavities, Phys. Rev. B: Condens. Matter Mater. Phys., 1997, 56, 429-433.

26 N. I. Cade, T. Ritman-Meer and D. Richards, Strong Coupling of Localized Plasmons and Molecular Excitons in Nanostructured Silver Films, Phys. Rev. B: Condens. Matter Mater. Phys., 2009, 79, 241404.

27 F. Nagasawa, M. Takase and K. Murakoshi, Raman Enhancement via Polariton States Produced by Strong Coupling between a Localized Surface Plasmon and Dye Excitons at Metal Nanogaps, J. Phys. Chem. Lett., 2014, 5, 14-19.

28 H. Minamimoto, F. Kato, F. Nagasawa, M. Takase and K. Murakoshi, Electrochemical Control of Strong Coupling States between Localized Surface Plasmons and Molecule Excitons for Raman Enhancement, Faraday Discuss., 2017, 205, 261-269.

29 D. G. Lidzey, D. D. C. Bradley, M. S. Skolnick, T. Virgili, S. Walker and D. M. Whittaker, Strong Exciton-Photon Coupling in an Organic Semiconductor Microcavity, Nature, 1998, 395, 53-55.

30 P. Törmä and W. L. Barnes, Strong Coupling between Surface Plasmon Polaritons and Emitters, Rep. Prog. Phys, 2015, 78, 013901.

31 J. Flick, N. Rivera and P. Narang, Strong Light-Matter Coupling in Quantum Chemistry and Quantum Photonics, Nanophotonics, 2018, 7, 1479-1501.

32 A. Shalabney, J. George, H. Hiura, J. A. Hutchison, C. Genet, P. Hellwig and T. W. Ebbesen, Enhanced Raman Scattering from Vibro-Polariton Hybrid States, Angew. Chem., Int. Ed., 2015, 54, 7971-7975.

33 J. del Pino, J. Feist and F. J. Garcia-Vidal, Signatures of Vibrational Strong Coupling in Raman Scattering, J. Phys. Chem. C, 2015, 119, 29132-29137.

34 A. Strashko and J. Keeling, Raman Scattering with Strongly Coupled Vibron-Polaritons, Phys. Rev. A, 2016, 94, 023843.

35 A. Shalabney, J. George, J. Hutchison, G. Pupillo, C. Genet and T. W. Ebbesen, Coherent Coupling of Molecular Resonators with a Microcavity Mode, Nat. Commun., 2015, 6, 5981 .

36 J. P. Long and B. S. Simpkins, Coherent Coupling between a Molecular Vibration and Fabry-Pérot Optical Cavity to Give Hybridized States in the Strong Coupling Limit, ACS Photonics, 2015, 2, 130-136.

37 R. F. Ribeiro, L. A. Martínez-Martínez, M. Du, J. CamposGonzalez-Angulo and J. Yuen-Zhou, Polariton Chemistry: Controlling Molecular Dynamics with Optical Cavities, Chem. Sci., 2018, 9, 6325-6339.

38 J. George, A. Shalabney, J. A. Hutchison, C. Genet and T. W. Ebbesen, Liquid-Phase Vibrational Strong Coupling, J. Phys. Chem. Lett., 2015, 6, 1027-1031.

39 J. D. Erwin, M. Smotzer and J. V. Coe, Effect of Strongly Coupled Vibration-Cavity Polaritons on the Bulk Vibrational States within a Wavelength-Scale Cavity, J. Phys. Chem. B, 2019, 123, 1302-1306.
40 V. F. Crum, S. R. Casey and J. R. Sparks, Photon-Mediated Hybridization of Molecular Vibrational States, Phys. Chem. Chem. Phys., 2017, 20, 850-857.

41 M. Hertzog, P. Rudquist, J. A. Hutchison, J. George, T. W. Ebbesen and K. Bçrjesson, Voltage-Controlled Switching of Strong Light - Matter Interactions Using Liquid Crystals, Chem. - Eur. J., 2017, 23, 18166-18170.

42 R. M. A. Vergauwe, J. George, T. Chervy, J. A. Hutchison and A. Shalabney, Quantum Strong Coupling with Protein Vibrational Modes, J. Phys. Chem. Lett., 2016, 7, 4159-4164.

43 K. S. Menghrajani, H. A. Fernandez, G. R. Nash and W. L. Barnes, Hybridization of Multiple Vibrational Modes via Strong Coupling Using Confined Light Fields, Adv. Opt. Mater., 2019, 7, 1900403.

44 A. Thomas, L. Lethuillier-Karl, K. Nagarajan, R. M. A. Vergauwe, J. George, T. Chervy, A. Shalabney, E. Devaux, C. Genet, J. Moran and T. W. Ebbesen, Tilting a Ground-State Reactivity Landscape by Vibrational Strong Coupling, Science, 2019, 363, 615-619.

45 Y. Pang, A. Thomas, K. Nagarajan, R. M. A. Vergauwe, K. Joseph, B. Patrahau, K. Wang, C. Genet and T. W. Ebbesen, On the Role of Symmetry in Vibrational Strong Coupling: The Case of Charge-Transfer Complexation, Angew. Chem., 2020, 132, 1-6.

46 A. Thomas, J. George, A. Shalabney, M. Dryzhakov, S. J. Varma, J. Moran, T. Chervy, X. Zhong, E. Devaux, C. Genet, J. A. Hutchison and T. W. Ebbesen, GroundState Chemical Reactivity under Vibrational Coupling to the Vacuum Electromagnetic Field, Angew. Chem., Int. Ed., 2016, 55, 11462-11466.

47 J. Lather, P. Bhatt, A. Thomas, T. W. Ebbesen and J. George, Cavity Catalysis by Cooperative Vibrational Strong Coupling of Reactant and Solvent Molecules, Angew. Chem., Int. Ed., 2019, 58, 10635-10638.

48 A. Thomas, A. Jayachandran, L. Lethuillier-Karl, R. M. A. Vergauwe, K. Nagarajan, E. Devaux, C. Genet, J. Moran and T. W. Ebbesen, Ground State Chemistry under Vibrational Strong Coupling: Dependence of Thermodynamic Parameters on the Rabi Splitting Energy, Nanophotonics, 2020, 9, 249-255.

49 K. Hirai, R. Takeda, J. A. Hutchison and H. Uji-i, Modulation of Prins Cyclization by Vibrational Strong Coupling, Angew. Chem., 2020, 132, 5370-5373.

50 B. Xiang, R. F. Ribeiro, M. Du, L. Chen, Z. Yang, J. Wang, J. Yuen-Zhou and W. Xiong, Intermolecular Vibrational Energy Transfer Enabled by Microcavity Strong Light, Science, 2020, 368, 665-667.

51 A. D. Dunkelberger, B. T. Spann, K. P. Fears, B. S. Simpkins and J. C. Owrutsky, Modified Relaxation Dynamics and Coherent Energy Exchange in Coupled Vibration-Cavity Polaritons, Nat. Commun., 2016, 7, 13504.

52 Z. Yang, B. Xiang and W. Xiong, Controlling Quantum Pathways in Molecular Vibrational Polaritons, ACS Photonics, 2020, 7, 919-924.

53 B. Xiang, R. F. Ribeiro, A. D. Dunkelberger, J. Wang, Y. Li, B. S. Simpkins, J. C. Owrutsky, J. Yuen-Zhou and W. Xiong, 
Two-Dimensional Infrared Spectroscopy of Vibrational Polaritons, Proc. Natl. Acad. Sci. U. S. A., 2018, 115, 4845-4850.

54 W. M. Takele, F. Wackenhut, L. Piatkowski, A. J. Meixner and J. Waluk, Multimode Vibrational Strong Coupling of Methyl Salicylate to a Fabry-Pérot Microcavity, J. Phys. Chem. B, 2020, 124, 5709-5716.

55 A. Chizhik, F. Schleifenbaum, R. Gutbrod, A. Chizhik, D. Khoptyar, A. J. Meixner and J. Enderlein, Tuning the Fluorescence Emission Spectra of a Single Molecule with a Variable Optical Subwavelength Metal Microcavity, Phys. Rev. Lett., 2009, 102, 073002.

56 M. Hertzog and K. Börjesson, The Effect of Coupling Mode in the Vibrational Strong Coupling Regime, ChemPhotoChem, 2020, 4, 612-617.

57 M. Hertzog, B. Munkhbat, D. Baranov, T. Shegai and K. Börjesson, Enhancing Vibrational Light-Matter Coupling Strength beyond the Molecular Concentration Limit Using Plasmonic Arrays, Nano Lett., 2021, 21, 1320-1326.

58 H. T. Varghese, C. Y. Panicker, D. Philip, J. R. Mannekutla and S. R. Inamdar, IR, Raman and SERS Studies of Methyl Salicylate, Spectrochim. Acta, Part A, 2007, 66, 959-963.

59 L. S. Taylor, F. W. Langkilde and G. Zografi, Fourier Transform Raman Spectroscopic Study of the Interaction of Water Vapor with Amorphous Polymers, J. Pharm. Sci., 2001, 90, 888-901.

60 K. B. R. Devi, K. B. Santhi and R. Mathivanan, Spectra and Normal Coordinate Analysis of Poly Vinyl Acetate, Eng. Sci. Technol., 2012, 6, 205-210.
61 C. Qian, Q. Guo, M. Xu, Y. Yuan and J. Yao, Improving the SERS Detection Sensitivity of Aromatic Molecules by a PDMS-Coated $\mathrm{Au}$ Nanoparticle Monolayer Film, RSC Adv., 2015, 5, 53306-53312.

62 J. Kapitán, L. Hecht and P. Bouř, Raman Spectral Evidence of Methyl Rotation in Liquid Toluene, Phys. Chem. Chem. Phys., 2008, 10, 1003-1008.

63 M. L. Patterson and M. J. Weaver, Surface-Enhanced Raman Spectroscopy as a Probe of Adsorbate-Surface Bonding: Simple Alkenes and Alkynes Adsorbed at Gold Electrodes, J. Phys. Chem., 1985, 89, 5046-5051.

64 J. George, T. Chervy, A. Shalabney, E. Devaux, H. Hiura, C. Genet and T. W. Ebbesen, Multiple Rabi Splittings under Ultrastrong Vibrational Coupling, Phys. Rev. Lett., 2016, 117, 153601.

65 T. W. Ebbesen, Hybrid Light-Matter States in a Molecular and Material Science Perspective, Acc. Chem. Res., 2016, 49, 2403-2412.

66 I. Vurgaftman, B. S. Simpkins, A. D. Dunkelberger and J. C. Owrutsky, Negligible Effect of Vibrational Polaritons on Chemical Reaction Rates via the Density of States Pathway, J. Phys. Chem. Lett., 2020, 11, 3557-3562.

67 R. Houdré, R. P. Stanley and M. Ilegems, Vacuum-field Rabi splitting in the presence of inhomogeneous broadening: Resolution of a homogeneous linewidth in an inhomogeneously broadened system, Phys. Rev. A: At., Mol., Opt. Phys., 1996, 53, 2711-2715. 